\title{
The present-day chemical composition of the LMC
}

\author{
W. R. J. Rolleston ${ }^{1}$, C. Trundle ${ }^{1}$, and P. L. Dufton ${ }^{1}$ \\ Department of Pure \& Applied Physics, The Queen's University of Belfast, BT7 1NN, Northern Ireland, UK \\ Received 5 June 2002 / Accepted 24 July 2002

\begin{abstract}
High-resolution observations of five OB-type main-sequence stars in the Large Magellanic Cloud (LMC) have been obtained with the UCL échelle spectrograph on the 3.9-m Anglo-Australian Telescope. These spectra have been analysed using LTE model-atmosphere techniques, to derive stellar atmospheric parameters and chemical compositions. As these stars are located within the hydrogen burning main-sequence band, their surface abundances should reflect those of the present-day interstellar medium. Detailed line-by-line differential analyses have been undertaken relative to Galactic comparison stars. We conclude that there exists a general metal deficiency of $-0.31 \pm 0.04$ dex within the LMC, and find no significant abundance variations between cluster and field stars. There is also tentative evidence to suggest a lower oxygen to iron abundance ratio, and an over-deficiency of magnesium relative to the other $\alpha$-elements. These are discussed in terms of previous abundance analyses and models of discontinuous (or bursting) star formation within the LMC. Finally, there is some evidence to suggest a greater chemical enrichment of material within the H II region LH 104.
\end{abstract}

Key words. stars: abundances - stars: atmospheres - stars: early-type - galaxies:individual: Large Magellanic Cloud

\section{Introduction}

The chemical composition of the interstellar medium (ISM) within a galaxy provides a fossil record of both stellar and galactic evolution. The Magellanic Clouds present a unique laboratory for testing such evolutionary theories as 1) they can be viewed in their entirety, 2) the galaxies possess well established distance estimates, 3) the line-of-sight is unobscured by dust, and 4) the Clouds are sufficiently close to enable individual stars and nebulae to be studied in detail. Furthermore, the star formation histories and chemical evolution in the Magellanic Clouds are significantly different to those of the Galactic disk (see Pagel \& Tautvaišienè 1998).

The Magellanic Clouds have been the subject of extensive elemental abundance investigations in recent years, (see, for example, Westerlund 1997; Olszewski et al. 1996; Rolleston 1991 for a review). It is evident that the global environments of these dwarf, irregular galaxies are completely different to that of the Milky Way - with mean metal deficiencies relative to the Sun of -0.3 dex and -0.65 dex for the Large (LMC) and Small (SMC) Clouds respectively (Russell \& Bessell 1989). However, the abundance patterns and the homogeneity of the metallicity throughout the Clouds are less well established. For example, several authors have observed the oxygen to iron abundance ratio in the LMC to be lower by $\sim 0.2-0.3$ dex than that found in Galactic stars in the solar neighbourhood (Russell \& Dopita 1992; Barbuy et al. 1994). This contrasts with the normal, Galactic value of the $[\mathrm{O} / \mathrm{Fe}]$ abundance ratio

Send offprint requests to: W. R. J. Rolleston,

e-mail: R.Rolleston@qub.ac.uk reported by Andrievsky et al. (2001) for a sample of LMC F-type supergiants. The determination of the $[\mathrm{O} / \mathrm{Fe}]$ ratio provides information about the relative contributions of type II to type I supernovae in the chemical enrichment of the LMC. Additionally, early studies of young, populous clusters in the Magellanic Clouds yielded metallicities that were significantly lower (by $\sim 0.5 \mathrm{dex}$ ) than the surrounding field populations. For example, Richtler et al. (1989) reported an iron underabundance of 0.9 dex for a red supergiant in the LMC cluster NGC 1818, and Spite et al. (1991) observed the iron-peak elements to be deficient by $\sim 1.0$ dex for similar objects in the SMC cluster NGC 330. However, more recent studies have reported smaller discrepancies between clusters and the field (see, for example, Korn et al. 2000).

Abundance determinations in the Clouds have relied mainly upon observations of emission-line plasmas, viz. H II regions and supernova remnants (Dufour 1984; Russell \& Dopita 1990), or of supergiants (Russell \& Bessell 1989; Spite et al. 1991). Such objects are not ideal for studying the current chemical composition of the ISM. For example, emission-line studies may be affected by the fractionation of some elements from the gas phase onto dust grains, whereas supergiant atmospheres may be contaminated by the products of nucleosynthesis that have been dredged up from the stellar interiors. Main-sequence B-type stars provide an excellent method for probing the metallicity and recent evolutionary history within the Milky Way and the Magellanic Clouds. Such stars have evolutionary ages typically less than $30 \mathrm{Myr}$ and possess photospheres that are not normally contaminated by core processed material (see Gies \& Lambert 1992). Furthermore, B-type dwarfs exhibit relatively 
rich, photospheric, metal-line spectra with absorption features due to $\mathrm{C}, \mathrm{N}, \mathrm{O}, \mathrm{Mg}, \mathrm{Si}, \mathrm{S}$ and $\mathrm{Fe}$. Hence, these objects should provide unambiguous information on the present-day chemical composition of the ISM within the parent galaxy.

We have previously reported reliable differential abundances for main-sequence B-types in the SMC (Rolleston et al. 1993; Lennon et al. 1996), LMC (Rolleston et al. 1996; hereafter, RBDH96), and the Inter-Cloud Region (Rolleston et al. 1999a). In this paper, we present results for an additional 3 LMC B-type main-sequence stars. Furthermore, we have reanalysed the spectra of the two objects published in RBDH96 using revised model-atmosphere techniques. Together, they represent a significant and homogeneous dataset of 5 unevolved stars distributed throughout the LMC.

\section{Observations and data reductions}

The spectroscopic data presented in this paper was obtained during seven observing campaigns - using various combinations of échelle and single order spectrographs, and both Image Photon Counting System (IPCS) and CCD detectors. Details of the epoch of observations are summarized in Table 1. Below we discuss the different telescope/instrumental configurations in more detail.

\subsection{AAT RGO spectrograph}

The new high-resolution spectroscopy presented in this paper (for LH 9-1160, LH 10-3270 \& LH 104-39) was primarily obtained with the 3.9-m Anglo-Australian Telescope (AAT) and the Royal Greenwich Observatory spectrograph (Stathakis \& Johnston 1997). The latter was mounted at Cassegrain $f / 8$ and operated with the $82 \mathrm{~cm}$ camera (blaze-to-collimator), the R1200B grating and a Tek $1 \mathrm{~K}$ CCD. This instrumental configuration yielded a full-width-half-maximum (FWHM) resolution of approximately $0.6 \AA$. Two wavelength settings were obtained, viz. $\lambda \lambda 3910-4155 \AA$ and $\lambda \lambda 4460-4705 \AA$, in order to observe the most important diagnostic lines for the determination of the stellar atmospheric parameters and photospheric abundances (see Sect. 3). Additionally, the blue spectral region facilitated the observation of interstellar absorption gas in the $\mathrm{Ca} \mathrm{H} \& \mathrm{~K}$ lines. Stellar observations were sub-divided into $1200 \mathrm{~s}$ integrations, so as to minimize the effects of cosmic ray events, and these were bracketed by copper-argon arc exposures for wavelength calibration. Bias frames and flat-field exposures, the latter made using a quartz continuum lamp, were taken at the beginning and end of each night.

\subsection{AAT UCLES spectroscopy}

Additional échelle spectroscopy of LH 104-39 was acquired using the AAT/UCLES, the 31 lines $\mathrm{mm}^{-1}$ grating and a Tek CCD in September 1996. Complete spectral coverage between $\lambda \lambda 3900-4900 \AA$ was achieved at a FWHM resolution of $\sim 0.1 \AA$ and at a $S / N \sim 30$ in the stellar continuum.

The observational data of the two stars presented in RBDH96 (viz. PS 34-16 \& LH 104-24) were obtained during two observing runs with the AAT in November 1989 and
December 1990. Stellar spectra were obtained with UCLES, the 79 lines $\mathrm{mm}^{-1}$ grating and an IPCS detector. The spectrograph was configured to provide a wavelength coverage from $\lambda \lambda 3920-4720 \AA$ (orders 57-48) at a FWHM resolution of $\sim 0.1 \AA$. Complete spectral coverage was not possible, and so the échelle was positioned to optimize the observation of the most important diagnostic lines. Further details can be found in RBDH96.

\subsection{SAAO spectrophotometry}

Spectrophotometry of the programme stars LH 9-1160 and LH 10-3270 was obtained using the SAAO 1.9-m telescope and cassegrain spectrograph during December 1994. The combination of Grating $6\left(100 \AA \mathrm{mm}^{-1}\right)$ and the Reticon Photon Counting System (RPCS) provided a FWHM resolution of $\sim 3.5 \AA$ and a useful spectral coverage between approximately $\lambda \lambda 3550-5500 \AA$. The observations were performed in photometric conditions and these were bracketed with exposures of flux standards, viz. L 870-2, LTT 2415, EG 158, LTT 9239 and LTT 1020.

\subsection{Comparison stars}

High signal-to-noise échelle spectroscopy of the bright, normal Population I B-type stars (viz. HR 1886, HR 2387 and 10 Lac) have been obtained previously with the $0.9-\mathrm{m}$ coudé feed telescope at Kitt Peak National Observatory. These data provide a spectral coverage of $\lambda \lambda 3900-4700 \AA$ at a FWHM resolution of $0.1 \AA$. Stellar spectra were again extracted using similar techniques, and equivalent width estimates for the non-diffuse helium and metal-lines have been published by Hambly et al. (1997).

Additionally, new AAT/UCLES CCD spectra of the Galactic comparison star $\tau$ Sco (HR 6165) was obtained in October 1999 using a similar instrumental configuration to that used in September 1996 (see Sect. 2.2).

\subsection{Reductions}

Details of the reduction procedures undertaken for the AAT/IPCS images can be found in RBDH96.

The two dimensional CCD datasets were reduced using standard procedures within IRAF (Tody 1986). Preliminary processing of the CCD frames such as overscan correction, trimming of the data section and flat-fielding were performed using the CCDRED package (Massey 1997), whilst cosmic-ray removal, extraction of the stellar spectra, sky subtraction and wavelength calibration were undertaken using tasks within the SPECRED (Massey et al. 1992) and DOECSLIT (Willmarth \& Barnes 1994) packages. Flux calibration of the SAAO spectrophotometry required two additional steps, an extinction correction and an instrumental sensitivity calibration, and these were applied within the DosLit task (Valdes 1992).

After co-addition of the extracted spectra from the individual image frames, a signal-to-noise ratio of $\sim 60-70$ was typically obtained in the stellar continua. Further manipulation of 
Table 1. Observational details of the programme stars.

\begin{tabular}{lccccccccc}
\hline \hline Object & \multicolumn{2}{c}{$\begin{array}{c}\delta \\
(2000.0)\end{array}$} & $V$ & $B-V$ & $E(B-V)$ & $\begin{array}{c}\text { AAT/ } \\
\text { UCLES }\end{array}$ & $\begin{array}{c}\text { AAT/ } \\
\text { RGO }\end{array}$ & $\begin{array}{c}\text { SAAO/ } \\
\text { RPCS }\end{array}$ & $\begin{array}{c}\text { Ref. } \\
\text { LH 9-1160 }\end{array}$ \\
\hline 04 56 32.9 & -662853 & 14.77 & -0.18 & 0.05 & - & $03 / 12 / 95$ & $12 / 12 / 94$ & 1 \\
LH 10-3270 & 045721.1 & -662501 & 14.90 & -0.24 & 0.10 & - & $03 / 12 / 95$ & $12 / 12 / 94$ & 1 \\
PS 34-16 & 050432.5 & -662447 & 15.00 & -0.15 & 0.07 & $08-10 / 11 / 89$ & - & - & 2 \\
& & & & & & $23-25 / 12 / 90$ & & - & 3 \\
LH 104-24 & 054005.9 & -692403 & 13.94 & -0.10 & 0.23 & $23-25 / 12 / 90$ & - & - & 3 \\
LH 104-39 & 054002.6 & -692524 & 15.40 & -0.06 & 0.23 & $26 / 09 / 96$ & $04-05 / 12 / 95$ & - & 3 \\
\hline
\end{tabular}

REFERENCES: - (1) Parker et al. (1992); (2) Shobbrook \& Visvanathan (1987); (3) Lucke (1974).

the stellar spectra, viz. radial velocity corrections, rectification of the stellar continuum and the measurement of equivalent widths for the metal and non-diffuse helium line-spectra, utilized the suite of routines provided by the spectral reduction package DIPso (Howarth et al. 1994). Full details of these methods can be found in Rolleston et al. (1999a) and references therein. The equivalent width data for the non-diffuse helium and metal lines can be obtained from the authors upon request.

\section{Data analysis}

The methods used to derive the stellar atmospheric parameters are similar to those discussed by Rolleston et al. (1999a, 2000). We have adopted theoretical results based on the ATLAs9 grid of line-blanketed model-atmospheres (Kurucz 1991) together with LTE radiative transfer codes. The model-atmospheres are characterized by four parameters, viz. effective temperature $\left(T_{\text {eff }}\right)$, surface gravity $(\log g)$, microturbulence $(\xi)$ and metallicity. Determination of these parameters is an iterative process - with all steps being inter-related and $T_{\text {eff }}, \log g$ and $\xi$ being dependent on metallicity which is not known a priori. It should be noted that the number of iterations were generally small, and that the adopted atmospheric parameters (see Table 2) were deduced using models with an appropriate chemical composition.

\subsection{Effective temperatures}

The classical method of deriving effective temperatures for early B-type stars utilizes ionization equilibria (viz. Si II/Si III, $\mathrm{Si}$ III/Si IV or $\mathrm{He}$ I/He II). Indeed, for the three stars with $T_{\text {eff }}>$ $29000 \mathrm{~K}$, it was possible to reliably measure absorption lines from two ionization stages of silicon ( $\mathrm{Si}$ III/Si IV). Hence, we adopted this technique as our primary temperature diagnostic. Furthermore, the optical spectra of LH 10-3270 and LH 10424 displayed absorption features due to the $\mathrm{He} / \mathrm{He}$ II ions. Balancing the latter ionization equilibrium yielded an effective temperature estimate within $1000 \mathrm{~K}$ of that derived using the silicon lines. Unfortunately, it was not possible to reliably extract profiles of the He II lines from the spectra of LH 10439 , due to the lower $S / N$ ratio that was obtained in the continuum. However, the neutral helium-line spectrum and the upper limit placed on the line-strength of the He II $4686 \AA$ feature are consistent with the $T_{\text {eff }}=30000 \mathrm{~K}$ deduced from the silicon ionization balance.

Due to the lower effective temperatures of LH 9-1160 and PS 34-16 see Table 2, absorption features due to He II or Si IV were not detected in the optical spectra. Furthermore, the combination of a B1 spectral type and the moderate rotation encountered for LH 9-1160 together with the lower $S / N$ ratio obtained with an IPCS detector for PS 34-16 precluded the observation of the Si II spectrum in both objects. Hence, it was necessary to use an alternative method to estimate the stellar effective temperatures. Shobbrook \& Visvanathan (1987) presented Strömgren photometry for 36 B-type stars in the LMC including PS 34-16. An effective temperature estimate was obtained for the latter from the reddening free $[u-b]$ index using the calibration of Napiwotzki et al. (1993). For LH 9-1160, we derived an effective temperature from a comparison of modelatmosphere calculations with the observed flux calibrated spectra obtained at SAAO. The latter was corrected for the effects of interstellar reddening using the $E(B-V)$ value of Parker et al. (1992) and an interstellar extinction law appropriate to the LMC (Howarth 1983). The slope of the stellar continuum is sensitive to the colour excess (over the observed wavelength range); typically, a change of $0.05 \mathrm{mag}$ in $E(B-V)$ corresponds to a change of approximately $1000 \mathrm{~K}$ in the derived stellar effective temperature. However, it is the Balmer discontinuity which is the most sensitive temperature diagnostic for these early-type stars and therefore, we fitted the stellar continuum around this feature.

Although different methods have been adopted for the determination of the stellar effective temperatures in the LMC targets - the strength of our abundance analyses/conclusions is founded in our careful differential approach. For each LMC programme star, we have selected a normal, Population I Galactic analogue which possesses similar atmospheric parameters $\left(T_{\text {eff }}, \log g, \xi\right)-$ see Table 2 . Effective temperature estimates for the latter were derived using an identical method to that adopted for the programme star.

\subsection{Surface gravities}

Surface gravities were deduced by fitting theoretical profiles to the normalized AAT spectra of the $\mathrm{H} \epsilon, \mathrm{H} \delta$ and $\mathrm{H} \gamma$ lines; where 
Table 2. Stellar parameters.

\begin{tabular}{lcccccccccc}
\hline \hline Star & $\begin{array}{c}T_{\text {eff }} \\
(\mathrm{K})\end{array}$ & $\begin{array}{c}\log g \\
(\mathrm{dex})\end{array}$ & $\begin{array}{c}\xi \\
\left(\mathrm{km} \mathrm{s}^{-1}\right)\end{array}$ & $\begin{array}{c}v \sin i \\
\left(\mathrm{~km} \mathrm{~s}^{-1}\right)\end{array}$ & $\begin{array}{c}\text { Mass } \\
\left(M_{\odot}\right)\end{array}$ & $\begin{array}{c}\text { Age } \\
(\mathrm{Myr})\end{array}$ & Comparison & $\begin{array}{c}T_{\text {eff }} \\
(\mathrm{K})\end{array}$ & $\begin{array}{c}\log g \\
(\mathrm{dex})\end{array}$ & $\begin{array}{c}\xi \\
\left(\mathrm{km} \mathrm{s}^{-1}\right)\end{array}$ \\
\hline LH 9-1160 & 25500 & 3.6 & 10 & 150 & 12.1 & 11.6 & HR 2387 & 26900 & 3.9 & 10 \\
LH 10-3270 & 29500 & 4.1 & 6 & 30 & 11.8 & 5.6 & HR 6165 & 30500 & 4.15 & 6 \\
LH 104-39 & 30000 & 4.4 & 5 & 13 & 10.5 & 0.6 & HR 6165 & 30500 & 4.15 & 6 \\
LH 104-24 & 34000 & 4.4 & 9 & 65 & 13.9 & 0.4 & HR 8622 & 35000 & 4.5 & 10 \\
PS 34-16 & 23000 & 4.0 & 5 & 40 & 7.5 & 17.9 & HR 1886 & 23500 & 4.1 & 6 \\
\hline
\end{tabular}

the theoretical calculations have adopted the line-broadening theory of Vidal et al. (1973).

\subsection{Microturbulence}

In early B-type stars, microturbulent velocities $(\xi)$ can be determined from, for example, their $\mathrm{O}$ II line-spectra by removing the dependence of the derived oxygen abundance upon the observed line-strengths (see Lennon et al. 1988). For LH 103270 and PS 34-16, this technique yielded values of 5 and $6 \mathrm{~km} \mathrm{~s}^{-1}$ respectively, in good agreement with the canonical value of $5 \mathrm{~km} \mathrm{~s}^{-1}$ that has been found previously from similar LTE analyses of B-type main-sequence stars (Hardorp \& Scholz 1970; Dufton et al. 1990). Unfortunately, the intrinsic weakness of the metal-line spectra in combination with the moderate rotation observed for LH 9-1160 and the lower $S / \mathrm{N}$ ratio obtained in the stellar continuum for LH 104-39 precluded the measurement of equivalent-width estimates for a sufficient number of $\mathrm{O}_{\text {II }}$ features in order to reliably determine the microturbulence parameter. Therefore, we have adopted our estimate for the microturbulent velocity of the corresponding standard star in the analysis of the programme stars. This seems reasonable, given that we have selected Galactic comparison stars that display very similar atmospheric parameters to that of the LMC stars.

A microturbulent velocity of $9 \mathrm{~km} \mathrm{~s}^{-1}$ was determined for LH 104-24 from 20 O II lines; this is somewhat greater than that found previously for B-type dwarfs (see above). However, the spectrum of this object (and the derived value of $T_{\text {eff }}$ ) is probably more compatible with a $09.5 \mathrm{v}$ than a B0v spectral type. Indeed, our Galactic comparison star is the 09.5v standard HR 8622 ( $\equiv 10 \mathrm{Lac}$ ); for which the O II line-spectrum yielded a value of $\xi=10 \mathrm{~km} \mathrm{~s}^{-1}$. Hence, we believe that this is justification for our choice of $\xi$ in the analysis of LH 104-24.

\subsection{Stellar $v \sin i$}

Stellar projected rotational velocities $(v \sin i)$ were estimated by convolving theoretically generated spectra with rotational broadening functions until they matched the observations. It should be noted that this procedure included the effect of instrumental broadening. Our estimates are presented in Table 2.
Table 3. Properties of the LMC gas.

\begin{tabular}{lccc}
\hline \hline Object & $\begin{array}{c}v_{\mathrm{lsr}}^{*} \\
\left(\mathrm{~km} \mathrm{~s}^{-1}\right)\end{array}$ & $\begin{array}{c}v_{\mathrm{lsr}}^{\mathrm{CaK}} \\
\left(\mathrm{km} \mathrm{s}^{-1}\right)\end{array}$ & $\begin{array}{c}W_{\mathrm{CaK}} \\
(\mathrm{m \AA})\end{array}$ \\
\hline LH 9-1160 & +261 & +248 & 159 \\
LH 10-3270 & +197 & +260 & 167 \\
PS 34-16 & +273 & +269 & 125 \\
LH 104-24 & +258 & +262 & 176 \\
LH 104-39 & +244 & +239 & 185 \\
\hline
\end{tabular}

\subsection{LMC membership}

It is important to confirm that the programme stars are indeed associated with the LMC. Two criteria were used to confirm their membership, viz. stellar radial velocities, and interstellar absorption lines. Radial velocities relative to the local standard of rest $\left(v_{\mathrm{lsr}}\right)$ have been deduced from the high-resolution AAT spectra (see Table 3) and are consistent with the estimate of $261 \mathrm{~km} \mathrm{~s}^{-1}$ for the heliocentric radial velocity of the LMC (Luks \& Rohlfs 1992). Additionally, the interstellar Ca II H and $\mathrm{K}$ features were observed in the stellar spectra. For all the programme stars, it was possible to identify absorption components due to both Galactic intermediate-velocity clouds and gas associated with the LMC (see Wayte 1990). Details of the LMC interstellar gas are presented in Table 3. Hence, it would appear that our targets are indeed members of the Clouds.

\subsection{Photospheric abundances}

The adopted atmospheric parameters (listed in Table 2) were used to derive absolute LTE abundances for both the programme and comparison stars using procedures similar to those described in Rolleston et al. (1999a, 1999b). Detailed line-byline differential abundances were also performed relative to the "normal" composition Galactic analogues HR 1886, HR 2387, HR 6165 and HR 8622. The validity for using these bright Galactic disk, B-type stars as standards of Population I chemical composition has been verified by Hambly et al. (1997).

\section{Discussion}

\subsection{Chemical composition of the LMC stars}

The model-atmosphere analyses are summarized in Tables 4-7 - the first presenting absolute abundances on a logarithmic 
Table 4. Absolute abundances of the LMC stars.

\begin{tabular}{|c|c|c|c|c|c|c|c|c|c|c|c|}
\hline Species & \multicolumn{2}{|c|}{$\begin{array}{l}\text { LH 9-1160 } \\
\end{array}$} & \multicolumn{2}{|c|}{ LH 10-3270 } & \multicolumn{2}{|c|}{ LH 104-39 } & \multicolumn{2}{|c|}{ "LH 104-24 } & \multicolumn{2}{|c|}{ PS 34-16 } & B-stars \\
\hline He I & $10.80 \pm 0.19$ & $(4)$ & $10.78 \pm 0.21$ & $(5)$ & $10.73 \pm 0.14$ & (7) & $11.03 \pm 0.07$ & $(6)$ & $10.82 \pm 0.23$ & (6) & 11.00 \\
\hline He II & - & & $10.99 \pm 0.32$ & (2) & $<11.00$ & (1) & 10.75 & (1) & - & & \\
\hline C II & - & & $<7.64$ & (1) & $<7.31$ & (1) & 7.56 & (1) & 7.03 & (1) & 8.20 \\
\hline C III & - & & 8.20 & (1) & $8.09 \pm 0.08$ & (3) & $7.44 \pm 0.32$ & (3) & - & & \\
\hline $\mathrm{N}_{\text {II }}$ & $7.40 \pm 0.21$ & (2) & $7.86 \pm 0.05$ & (2) & $<7.65$ & (1) & 7.76 & (1) & $7.66 \pm 0.09$ & (4) & 7.81 \\
\hline N ini & - & & $8.25 \pm 0.03$ & (2) & - & & 7.62 & (1) & - & & \\
\hline O II & $8.29 \pm 0.26$ & (13) & $8.28 \pm 0.14$ & (13) & $8.45 \pm 0.32$ & (13) & $8.59 \pm 0.19$ & (23) & $8.65 \pm 0.24$ & (18) & 8.68 \\
\hline Mg II & 6.93 & (1) & 6.81 & (1) & $<7.15$ & (1) & 7.44 & (1) & 6.72 & (1) & 7.38 \\
\hline Si III & $6.98 \pm 0.12$ & (3) & $7.21 \pm 0.06$ & (3) & 7.32 & (1) & $7.78 \pm 0.01$ & (2) & $7.11 \pm 0.08$ & (2) & 7.58 \\
\hline Si IV & - & & 7.22 & (1) & 7.38 & (1) & 7.84 & (1) & - & & \\
\hline S III & - & & - & & - & & 6.25 & (1) & - & & 7.21 \\
\hline Fe III & - & & - & & - & & - & & $6.89 \pm 0.60$ & (2) & 7.72 \\
\hline
\end{tabular}

1. Errors (where quoted) refer to the $1 \sigma$ standard deviation of abundance estimates obtained from individual lines. Uncertainties on the single measurements should be typically less than 0.2 dex (see Sect. 3.1).

2. The numbers in parentheses after each abundance estimate represent the number of absorption lines used in the model-atmosphere analysis.

3. Normal, Population I B-star values have been taken from Gies \& Lambert (1992) - non-LTE abundances for He, C, N, O, \& Si, and Kilian (1994), viz. non-LTE abundance for Mg and LTE abundances for S \& Fe.

scale with $[\mathrm{H}] \equiv 12.0$ dex, the others listing differential abundances again on a logarithmic scale; errors (where quoted) refer to the $1 \sigma$ sample standard deviations. A typical observational uncertainty of $\pm 10 \mathrm{~m} \AA$ in the equivalent-width estimate of an individual line would lead to an abundance error of \pm 0.15 dex. Additionally, the effect of changing the atmospheric parameters by their error estimates was considered, viz. $\Delta T_{\text {eff }} \sim \pm 1000 \mathrm{~K}$, $\triangle \log g \sim \pm 0.2$ dex and $\Delta \xi \sim \pm 3 \mathrm{~km} \mathrm{~s}^{-1}$, and led to changes in the absolute abundance estimates by less than 0.2 dex. For the differential analyses, the effect of systematic errors in the estimates of the atmospheric parameters will be reduced, while uncertainties in the adopted atomic data will also be less important. Additionally, simplifications in the model-atmosphere analyses (e.g. the assumption of LTE) should also be less significant for the differential abundances.

The absolute abundances for the five LMC stars are given in Table 4. For comparison purposes, we have listed the mean values reported by Gies \& Lambert (1992) and Kilian (1994) for unevolved, early B-type stars found in the local field and OB associations. However, the photospheric abundances of our LMC targets have been derived using different equivalent-width datasets, different atomic data and different model-atmospheres to those used in the Gies \& Lambert and Kilian investigations. Hence, for these reasons and those discussed above, it is more instructive to compare the differential abundances presented in Table 5. The number of lines used to derive the differential abundances are given in parentheses after each estimate. Additionally, we list in the last column of Table 5 the mean values of the stellar abundance estimates derived for each species.

\subsubsection{Helium}

Absolute abundances derived for our five programme stars are similar to that found for normal, Population I, Galactic, B-type main-sequence stars. Indeed, the differential abundances imply that there are no significant differences between the helium content of the LMC stars and their Galactic analogues. This provides indirect evidence that the atmospheres of our LMC targets have not been contaminated by nuclear processed material mixed from their interiors and hence should be reliable tracers of the chemical composition of their natal environment.

\subsubsection{Carbon}

An inspection of Table 4 would suggest significant differences between the absolute photospheric carbon compositions (derived using C III lines) of LH 10-3270/LH 104-39 ( 8.1 dex) and LH 104-24 ( 7.4 dex). However, these abundance differences are almost certainly attributable to temperature sensitive non-LTE effects and the effective temperature of LH 104-24 being $\sim 4000 \mathrm{~K}$ hotter than that of LH 10-3270 and LH 10439 (see Table 2). Indeed, the scatter in the differential abundances of the three objects is only 0.3 dex. Furthermore, it is the hottest star, viz, LH 104-24 with the lowest absolute C III abundance estimate $(\sim 7.4 \mathrm{dex})$, which yields the smallest carbon deficiency of $\sim 0.26 \mathrm{dex}$. The above illustrates the importance of undertaking detailed line-by-line differential abundance analyses relative to normal, Galactic comparison stars of similar atmospheric parameters - and that absolute LTE abundances should be treated with caution. We conclude that there are no significant differences in the photospheric carbon abundance of our stars.

\subsubsection{Nitrogen}

Abundance estimates for nitrogen were primarily derived using the $\mathrm{N}$ II line-spectra. The differential analyses yield a large spread in nitrogen compositions - ranging from -0.5 dex in 

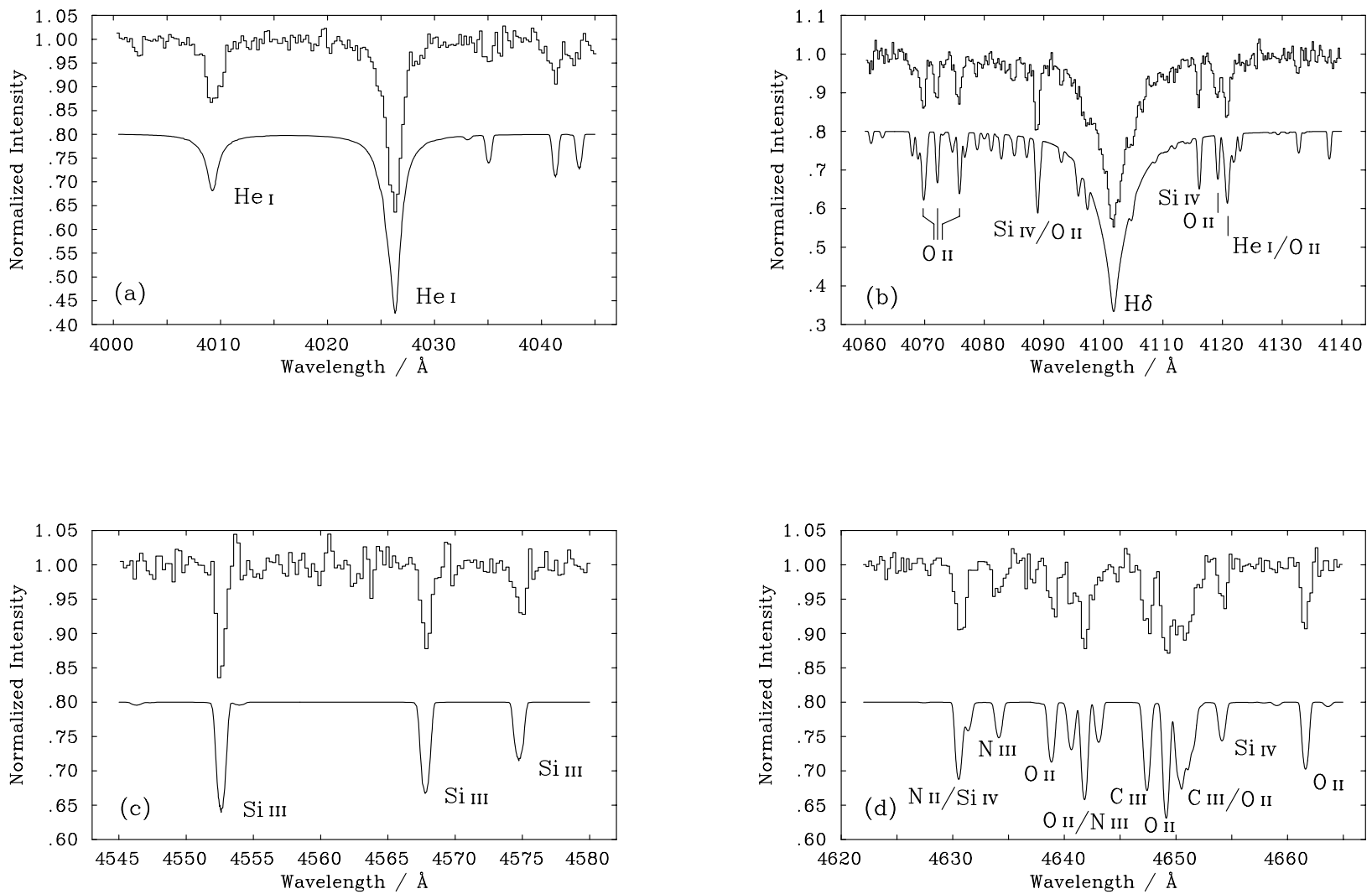

Fig. 1. Examples of the observed spectra (histograms) obtained for LH 10-3270 using the RGO spectrograph on the AAT, for spectral regions including a) neutral helium features, b) $\mathrm{H} \delta$ profile, and c), d) numerous metal lines. The synthesized spectra (smooth curves) have been generated using the adopted atmospheric parameters derived for LH 10-3270 (see Table 2) and the absolute abundances presented in Table 4. Excellent agreement is found between observation and theory.

Table 5. Differential abundance analysis.

\begin{tabular}{lccccccccccc}
\hline \hline Species & LH 9-1160 & \multicolumn{2}{c}{ LH 10-3270 } & \multicolumn{2}{c}{ LH 104-39 } & \multicolumn{2}{c}{ LH 104-24 } & \multicolumn{2}{c}{ PS 34-16 } & Mean \\
\hline He I & $+0.15 \pm 0.04$ & $(3)$ & $-0.12 \pm 0.06$ & $(4)$ & $-0.21 \pm 0.19$ & $(7)$ & $-0.17 \pm 0.15$ & $(6)$ & $+0.01 \pm 0.16$ & $(5)$ & $-0.07 \pm 0.15$ \\
He II & - & & $+0.06 \pm 0.01$ & $(2)$ & $<-0.20$ & $(1)$ & -0.25 & $(1)$ & - & & $-0.10 \pm 0.22$ \\
C II & - & & $<-0.11$ & $(1)$ & $<-0.32$ & $(1)$ & -0.27 & $(1)$ & -0.27 & $(1)$ & $-0.27 \pm 0.01$ \\
C III & - & & -0.40 & $(1)$ & $-0.47 \pm 0.04$ & $(1)$ & $-0.26 \pm 0.08$ & $(3)$ & - & & $-0.38 \pm 0.11$ \\
N II & $-0.49 \pm 0.13$ & $(2)$ & $-0.24 \pm 0.02$ & $(2)$ & $<-0.44$ & $(1)$ & -0.07 & $(1)$ & $+0.06 \pm 0.09$ & $(4)$ & $-0.18 \pm 0.24$ \\
N III & - & & $+0.04 \pm 0.03$ & $(2)$ & - & & -0.60 & $(1)$ & - & & $-0.28 \pm 0.45$ \\
O II & $-0.30 \pm 0.25$ & $(11)$ & $-0.32 \pm 0.12$ & $(13)$ & $-0.13 \pm 0.21$ & $(13)$ & $+0.07 \pm 0.14$ & $(18)$ & $-0.33 \pm 0.14$ & $(13)$ & $-0.20 \pm 0.17$ \\
Mg II & -0.40 & $(1)$ & -0.63 & $(1)$ & $<-0.36$ & $(1)$ & -0.26 & $(1)$ & -0.38 & $(1)$ & $-0.42 \pm 0.15$ \\
Si III & $-0.33 \pm 0.17$ & $(2)$ & $-0.31 \pm 0.07$ & $(3)$ & -0.17 & $(1)$ & $+0.04 \pm 0.03$ & $(2)$ & $-0.26 \pm 0.04$ & $(2)$ & $-0.21 \pm 0.15$ \\
Si IV & - & & -0.34 & $(1)$ & -0.19 & $(1)$ & +0.08 & $(1)$ & - & $-0.15 \pm 0.21$ \\
S III & - & & - & - & - & & -0.23 & $(1)$ & - & -0.23 \\
Fe III & - & & - & & - & & - & & $-0.17 \pm 0.06$ & $(2)$ & -0.17 \\
\hline
\end{tabular}

1. Error estimates are as discussed in Table 4.

2. The number in parentheses is the number of lines used in the differential abundance analysis.

3. The last column represents the mean of the differential abundance estimates obtained for all stars.

LH 9-1160 to +0.06 dex in PS 34-16. Indeed, the latter results for PS 34-16 would suggest that nitrogen is significantly enhanced in the photosphere of this star relative to the mean metallicity of approximately $-0.3 \mathrm{dex}$ as derived from the $\alpha$-elements oxygen and silicon (see Table 6). This may be the result of processed material being dredged up from the stellar interior (see Gies \& Lambert 1992). However, this object is located within the main-sequence band, it displays no corresponding helium enhancement nor carbon deficiency, and exhibits a low $v \sin i$ value.

Alternatively, processed material may have been accreted onto the photosphere of PS 34-16. For example, one could speculate that PS 34-16 is a runaway star - ejected from a LMC OB association through a binary supernova 
Table 6. Metallicity of individual stars

\begin{tabular}{lcc}
\hline \hline Star & $\Delta[m / \mathrm{H}]_{\mathrm{O}, \mathrm{Si}}$ & $\Delta[m / \mathrm{H}]_{\mathrm{O}, \mathrm{Mg}, \mathrm{Si}}$ \\
\hline LH 9-1160 & $-0.32 \pm 0.02$ & $-0.34 \pm 0.05$ \\
LH 10-3270 & $-0.32 \pm 0.01$ & $-0.42 \pm 0.18$ \\
LH 104-39 & $-0.15 \pm 0.03$ & - \\
LH 104-24 & $+0.06 \pm 0.02$ & $-0.05 \pm 0.18$ \\
PS 34-16 & $-0.30 \pm 0.05$ & $-0.32 \pm 0.06$ \\
\hline
\end{tabular}

1. $\Delta[m / \mathrm{H}]_{\mathrm{O}, \mathrm{Si}}=(\Delta[\mathrm{O} / \mathrm{H}]+\Delta[\mathrm{Si} / \mathrm{H}]) / 2$

2. $\Delta[m / \mathrm{H}]_{\mathrm{O}, \mathrm{Mg}, \mathrm{Si}}=(\Delta[\mathrm{O} / \mathrm{H}]+\Delta[\mathrm{Mg} / \mathrm{H}]+\Delta[\mathrm{Si} / \mathrm{H}]) / 3$

(BS) explosive event (see Blaauw 1961). In the BS model, a massive close binary system undergoes Roche Lobe overflow during which mass and angular momentum is transferred from the primary to the secondary. The former evolves into a helium star before exploding as a supernova. This provides an impulse to the system which results in a large space velocity of the secondary. During the Roche Lobe overflow phase, CNO processed material is transferred from the primary to the secondary, leading to an enhanced nitrogen composition. However, a comparison of the LSR velocities of PS 34-16 and the LMC gas along this sightline does not support a large peculiar motion; although, this is inconclusive in itself as we can not constrain the magnitude of any transverse space motion. Secondly, the BS ejection mechanism results in a significant transfer of angular momentum to the secondary which is not supported by the small value of $v \sin i \sim 40 \mathrm{~km} \mathrm{~s}^{-1}$ observed for PS 34-16. Hence, it is unclear whether this observed nitrogen enhancement is evidence for processed material being mixed into the photosphere of PS 34-16 or a reflection of an enhanced nitrogen composition within the progenitor ISM.

We also deduced nitrogen abundance estimates for LH 10-3270 and LH 104-24 from their N III line-spectra. For LH 10-3270, this estimate was in reasonable agreement with that derived from the two $\mathrm{N}_{\text {II }}$ features. However, the LH 104-24 differential abundances are discrepant by $\sim 0.5 \mathrm{dex}$ for the $\mathrm{N}$ II/ $/ \mathrm{N}$ III ions. Unfortunately, equivalent width estimates were only measured for one line from each ionization stage. Additionally, at the temperature of LH 104-24 ( $T_{\text {eff }} \sim$ $34000 \mathrm{~K})$ - neither feature is an ideal diagnostic of the nitrogen abundance. First, the N II line-spectrum is intrinsically weak as $\mathrm{N}$ III becomes the dominant ion at temperatures greater than $30000 \mathrm{~K}$ (see Becker \& Butler 1989). Indeed, the line-strength of the $\mathrm{N}_{\text {II }} 3995.00 \AA$ feature is only $W_{\lambda} \sim 30 \mathrm{~m} \AA$. Secondly, the strong $\mathrm{N}_{\text {III }}$ feature $\left(W_{\lambda} \sim 130 \mathrm{~m} \AA\right)$ is blended with an $\mathrm{O}_{\text {II }}$ line. It has been also known for some time that major departures from LTE play a dominant role in the formation of the $\mathrm{H}$ and $\mathrm{He}$ line-spectra in such a temperature regime (Auer \& Mihalas 1972). Furthermore, significant differences exist between the UV fluxes and metal-line equivalent widths predicted using LTE and non-LTE line-formation calculations (see, for example, Hubeny et al. 1998). Hence, we believe that our nitrogen abundance estimates for LH 104-24 should be treated with caution.

\subsubsection{Oxygen, silicon}

The $\mathrm{O}$ II line-spectrum was the best observed species in all of our LMC stars, with on average 14 lines contributing to the mean differential oxygen abundance. Reliable silicon abundances were also deduced from the Si III lines (Multiplet No. 2) located in the relatively line-free region of the spectrum, $\lambda \lambda 4552-4574 \AA$. Hence, abundance estimates for these two $\alpha$-elements provide a reliable value of the general metallicity of each star.

\subsubsection{Magnesium, sulphur, iron}

Estimates of the magnesium abundance are based on one line, viz. the $\mathrm{Mg}$ II doublet at $4481.2 \AA$; although, this feature was generally well observed in our spectra. Unfortunately, it was only possible to reliably measure equivalent widths for one S III line in LH 104-24 and two Fe III transitions in PS 34-16. Thus, abundance estimates for sulphur and iron should be treated with caution.

\subsubsection{General metallicity}

The mean abundances presented in Table 5 for each species appear to be consistent with the canonical values of the presentday, LMC metallicity found in the literature (see, for example, Russell \& Dopita 1992; Garnett 1995). However, our values have been calculated without giving consideration to possible spatial chemical inhomogeneities within the LMC. Therefore, we have deduced the mean metallicity of the individual stars using our abundance estimates for oxygen and silicon. These elements should be representative of the overall atmospheric metallicity as these objects are within (or in the case of LH 9-1160, close to) the main-sequence band. Indeed, it is difficult to envisage how the photospheric composition of $\mathrm{O}, \mathrm{Si}$ (or $\mathrm{Mg}$ ) could have been altered since the formation of our stars. In Table 6 , we present our estimates for the mean metallicity of each object where $\Delta[m / \mathrm{H}]_{\mathrm{O}, \mathrm{Si}}=(\Delta[\mathrm{O} / \mathrm{H}]+\Delta[\mathrm{Si} / \mathrm{H}]) / 2$. It should be noted that measuring the metallicity of the programme stars using the three $\alpha$-elements $(\mathrm{O}, \mathrm{Mg}, \mathrm{Si})$ made no difference to the following discussion and conclusions (see Table 6).

Three objects display very similar metallicities of -0.3 dex, viz. LH 9-1160, LH 10-3270 and the field star PS 34-16. The associations LH 9 and LH 10, located in the North-west corner of the LMC, belong to a group of $5 \mathrm{H}$ iा regions which show strong $\mathrm{H}_{\text {II }}$ emission, while the entire complex is embedded in a strong H I region (Lucke 1974). PS 34-16 is situated $\sim 7.5^{\prime}(\sim 660 \mathrm{pc}$ ) away from LH 9/LH 10. Hence, we conclude that there are no significant differences between the general metallicity of the field and OB associations/H II regions in this part of the LMC. Similarly, Korn et al. (2000) found no evidence for large differences between cluster and field B-type, bright giants in the Clouds. Indeed, the large deficiencies reported by Richtler et al. (1989) and Spite et al. (1991) for red supergiants in LMC/SMC clusters may be partially attributed to the underestimation of $\mathrm{MC}$ abundances, due to incorrectly assigning stellar parameters as a result 
Table 7. Summary of LMC Metallicity investigations.

\begin{tabular}{llccccc}
\hline \hline \multirow{2}{*}{ Element } & \multicolumn{5}{c}{$\Delta[\mathrm{X} / \mathrm{H}]$} \\
& This paper & R\&D92 & HAS95 & LMB98 & KBG00 & AKK01 \\
\hline $\mathrm{He}$ & $+0.04 \pm 0.09$ & -0.06 & - & - & - & - \\
$\mathrm{C}$ & $-0.34 \pm 0.09$ & -0.29 & -0.31 & -0.12 & -0.35 & -0.45 \\
$\mathrm{~N}$ & $-0.30 \pm 0.18$ & -0.43 & - & - & -0.03 & - \\
$\mathrm{O}$ & $-0.32 \pm 0.02$ & -0.35 & -0.18 & -0.23 & -0.19 & -0.45 \\
$\mathrm{Mg}$ & $-0.47 \pm 0.14$ & -0.10 & -0.34 & -0.35 & -0.54 & -0.67 \\
$\mathrm{Si}$ & $-0.30 \pm 0.04$ & -0.18 & -0.18 & -0.26 & -0.44 & -0.30 \\
$\mathrm{Fe}$ & -0.17 & -0.19 & -0.32 & -0.28 & -0.16 & -0.47 \\
\hline
\end{tabular}

R\&D92: Russell \& Dopita (1992) - elements C, Mg, Si and Fe were deduced from F-type supergiants relative to Canopus; He, N, O, S were obtained from $\mathrm{H}_{\text {II }}$ regions and supernovae remnants relative to Galactic objects (Russell \& Dopita 1990; Shaver et al. 1983).

HAS95: Hill et al. (1995) - abundance estimates were derived from 9 F-type supergiants relative to Canopus.

LMB98: Luck et al. (1998) - 9 LMC Cepheids analysed relative to Galactic analogue stars.

KBG00: Korn et al. (2000) - differential abundance estimates of 6 evolved, B-type giants/bright giants were derived from a comparison with B-type dwarfs in the solar neighbourhood (C, N, O, Mg and Si - Gummersbach et al. 1998; Fe - Kaufer et al. 1994).

AKK01: Andrievsky et al. (2001) - 9 F-type supergiants analysed relative to $\delta$ Cep (Kovtyukh \& Andrievsky 1999).

of uncertainties in the non-uniform reddening along these sight-lines.

By contrast, we observe a smaller metal deficiency of -0.15 dex for star-39 and a slight metal overabundance of +0.06 dex for star-24 in the H II region LH 104. It is interesting that both stars display a larger metallicity than that normally associated with the present-day LMC ISM. However, it should be noted that the observational dataset for star-39 was of lower quality (UCLES spectra: $S / N \sim 25$; RGO spectra: $S / N \sim 50$ in the stellar continuum). Consequently, the abundance estimates are based on a smaller subset of lines. Star-24 has an effective temperature of $34000 \mathrm{~K}$ - comparable with a $09.5 \mathrm{v}$ spectral type - where non-LTE effects become significant (see Sect. 4.1.3). However, a direct comparison of the hydrogen/helium line-spectra for LH 104-24 and the Galactic star HR 8622 illustrates the very similar spectral types/luminosity classes (and hence atmospheric parameters) of both stars. Furthermore, the line-strengths of their silicon and oxygen spectra are also comparable (see Fig. 2). This provides unambiguous evidence that both objects have very similar photospheric silicon/oxygen compositions. Hence, we do not believe that the neglection of non-LTE effects can explain the normal metallicity found for LH 104-24.

LH 104 and five other OB associations, viz. LH 101, LH 103, LH 105, LH 106 and LH 108, are contained within the giant H II region N 158 (Henize 1956) which is located $\sim 20^{\prime}$ south of 30 Doradus. The northern part of N 158 comprises a superbubble around the OB association LH 104. Testor \& Niemela (1998) have constructed H-R diagrams for the upper main-sequence of the two associations LH 101 and LH 104. These observations are consistent with the 2 objects having stellar populations of $2-6 \mathrm{Myr}$ and $\leq 2 \mathrm{Myr}$ respectively. This is also compatible with the spectroscopic studies of Schild \& Testor (1992) and Testor \& Niemela (1996) which revealed that LH 104 is dominated by B-stars and LH 101 by massive O-type stars. Furthermore, it is in good agreement with our age estimates of 0.4 Myr and 0.6 Myr for star-24 and star-39 respectively (see Table 2). Given that this region is an active site of very massive star formation - it is possible that the higher metallicity observed for LH 104-24 and LH 104-39 reflects a greater enrichment of the ISM in this region by previous generations of massive stars, whose deaths (evidenced by the superbubble) may have triggered this current epoch of star formation. However, we believe that further high-quality observations of B-type main-sequence stars in LH 104 are required before a definitive conclusion can be made.

\subsection{Present-day metallicity of the $L M C$}

In Table 7, we present our best estimate for the present-day global metallicity of the ISM within the LMC. These mean values were obtained using the results of the abundance analysis presented in Table 5, and excluded the results obtained for the 2 stars associated with the $\mathrm{H}_{\text {II }}$ region LH 104, as it is unclear whether this region has experienced extraordinary epochs of massive star formation (see Sect. 4.1.6). However, it should be noted that in general their inclusion would not affect the abundance estimates presented in Table 7 . Indeed, the greatest effect is observed for the elements oxygen and silicon where the inclusion of abundance estimates for the two LH 104 stars would change the mean values by 0.1 dex. Additionally, PS 3416 displays a nitrogen enhancement which may be evidence of $\mathrm{CN}$-cycle material having been mixed into the photosphere (see Sect. 4.1.3); hence, this object was not included in the calculation of the general nitrogen composition.

It is important to compare our results for young, mainsequence stars with abundance estimates obtained for $\mathrm{H}$ II regions and more luminous, more evolved supergiants. However, a direct comparison of absolute abundance estimates is probably not reliable as different classes of objects utilize different modelling techniques. A perusal of the published stellar analyses alone illustrates the range of model-atmosphere codes, 


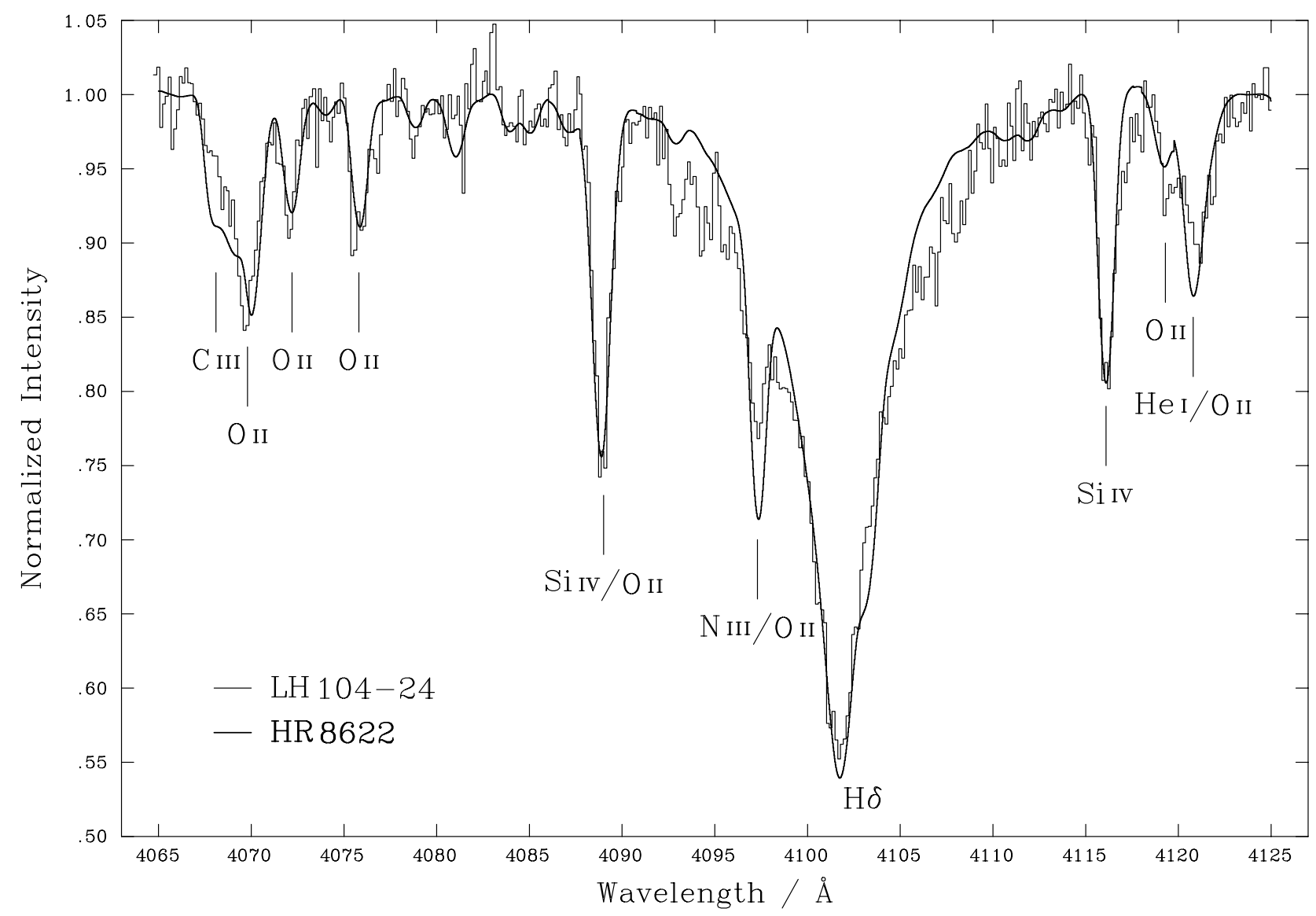

Fig. 2. Part of the observed spectrum including the H $\delta$ region for the LMC star LH 104-24 and the Galactic star HR 8622. The latter spectrum has been convolved with a rotational broadening function so that it matches the observed $v \sin i$ value of $65 \mathrm{~km} \mathrm{~s}^{-1}$ observed for LH 104-24. The close similarity between the hydrogen/helium line-spectra illustrates the very similar spectral types/luminosity classes (atmospheric parameters) of both stars. Comparable line-strengths are observed for the Si IV and $\mathrm{O}$ II features - providing unambiguous evidence that both objects have very similar photospheric silicon/oxygen compositions. Furthermore, the $\mathrm{C}$ III lines at $\sim 4068 \AA$ and the $\mathrm{N}$ III $4097.3 \AA$ feature are weaker in the LMC star - consistent with carbon and nitrogen being deficient in LH 104-24 (see Table 5).

different atomic/molecular transitions, and sources of atomic data used to deduce elemental abundances for the LMC (see, for example, Russell \& Bessell 1989; Hill et al. 1995; Luck et al. 1998; Korn et al. 2000). This in turn may lead to systematic differences between the sets of results. By the same rationale, abundance ratios defined relative to the solar values, ie. $[X / \mathrm{H}]=\log (X / \mathrm{H})_{\text {object }}-\log (X / \mathrm{H})_{\odot}$ may not be directly comparable. For example, a model-atmosphere analysis of the bright, Galactic, F-type supergiant Canopus has been performed by several authors. Estimates of the iron abundance ratio $[\mathrm{Fe} / \mathrm{H}]$ differ by upto $0.3 \mathrm{dex}$, viz. $-0.12 \mathrm{dex}$ (Russell \& Bessell 1989), +0.06 dex (Hill et al. 1995) \& -0.22 dex (Luck et al. 1998). It is unlikely that these discrepancies are the result of observational uncertainties, as the visual magnitude of Canopus is $V=-0.7$. Rather, the range of iron abundance estimates is most likely the consequence of systematic differences between the model-atmosphere techniques. Unfortunately, the magnitude of such differences are as large as the observed general metallicity of the LMC; hence, conclusions based upon the direct comparisons between sets of absolute abundance estimates are limited in their usefulness.
However, such problems can be easily circumvented if the LMC objects are analysed relative to similar objects in the solar neighbourhood using the same modelling techniques (see, for example, Russell \& Dopita 1992). We have previously illustrated the merits of undertaking a detailed differential approach (see, for example, Rolleston et al. 2000). Hence, we have attempted to apply our prescription of a detailed differential analysis to a selection of representative LMC studies, viz. H II regions (Russell \& Dopita 1992), F-type supergiants (Hill et al. 1995; Andrievsky et al. 2001), Cepheids (Luck et al. 1998) and B-type giants/bright giants (Korn et al. 2000). Using the published absolute abundances (or abundance ratios $[X / \mathrm{H}]$ ) for both the LMC targets and similar objects in the solar neighbourhood, we have computed differential abundances, $\Delta[X / \mathrm{H}]=\log (X / H)_{\mathrm{OBJECT}}-\log (X / \mathrm{H})_{\mathrm{GALACTIC}}$, which should be directly inter-comparable with our results. The compilation is presented in Table 7. Unfortunately, not all authors published abundance information for Galactic comparison objects. Galactic analogues for the sample of B-type, bright giants (Korn et al. 2000) were adopted from similar modelatmosphere analyses of B-type dwarfs in the solar vicinity 
published by the same Heidelberg group, viz. Gummersbach et al. (1998) for C, N, O, Mg, and Si and Kaufer et al. (1994) for Fe. The F-type Cepheid $\delta$ Cep (Kovtyukh \& Andrievsky 1999) was adopted as a comparison for the 9 LMC F-type supergiants published by Andrievsky et al. (2001); both sets of analyses were performed using the same revised model-atmosphere methods.

Inspection of Table 7 demonstrates that reasonable agreement is found for the overall metallicity of the LMC - irrespective of the class of object used to trace the chemical composition of the ISM. Indeed, the mean metallicity (as defined by $\Delta[M / \mathrm{H}]=(\Delta[\mathrm{O} / \mathrm{H}]+\Delta[\mathrm{Si} / \mathrm{H}]) / 2)$ obtained by the 6 analyses given in Table 7 is $-0.30 \pm 0.08$ dex. It should be noted that recent metallicity determinations of young, LMC clusters $([\mathrm{Fe} / \mathrm{H}]=-0.43 \mathrm{dex})$ and the field $([\mathrm{Fe} / \mathrm{H}]-0.44 \mathrm{dex})$ using Strömgren CCD photometry are in reasonable agreement with the spectroscopic investigations (see Hilker et al. 1995; Larsen et al. 2000). However, these photometric metallicities are absolute estimates measured relative to the Sun, and so may not be directly comparable to the results presented in Table 7. A metallicity of $-0.31 \pm 0.04$ dex is derived for the young, B-type dwarfs presented here. Additionally, the element abundances appear to be more tightly constrained around the mean value, than compared with the other analyses. Indeed, the only elements that may significantly deviate from the mean of -0.31 dex are magnesium and iron (see discussion below).

\subsection{1. $\triangle[\mathrm{N} / \mathrm{O}]$ abundance ratio}

Table 7 illustrates one advantage of using B-type stars as tracers of chemical composition - as they provide complete information about $\mathrm{CNO}$ abundance ratios. However, inspection of the $\Delta[\mathrm{N} / \mathrm{O}]$ abundance ratio illustrates the importance of studying objects that lie within the main-sequence band. This abundance ratio is effectively zero in our sample of dwarfs; furthermore, the individual estimates of nitrogen and oxygen are in good agreement with that obtained for $\mathrm{H}$ in regions. However, the products of hydrogen burning appear to have been mixed into the photospheres of the B-type giants/bright giants of the Korn et al. (2000) sample - as they exhibit a nitrogen enhancement, $\Delta[\mathrm{N} / \mathrm{O}] \sim+0.2$ dex.

\subsection{2. $\alpha$-elements $(\mathrm{O}, \mathrm{Mg}, \mathrm{Si})$}

Magnesium is underabundant relative to the other $\alpha$-elements $(\mathrm{O}, \mathrm{Si})$ in our sample of B-type dwarfs by approximately $0.16 \mathrm{dex}$. This result should be treated with caution, as the magnesium abundance is based on only one transition in each star. However, it is interesting to note that a similar abundance pattern, $\Delta[\mathrm{Mg} / \alpha]=-0.19 \pm 0.07 \mathrm{dex}$, is observed in the studies of evolved supergiants. Thus, there is some evidence to suggest that the LMC ISM is indeed more deficient in magnesium. A similar deficiency was observed in a sample of SMC B-type supergiants (see Dufton et al. 2000); although, the results are less conclusive for the dataset of SMC B-type dwarfs (see Rolleston et al. 1993). Unfortunately, no explanation has been offered for this tentative magnesium over-deficiency.
Type II supernovae are believed to be the dominant contributor of oxygen, magnesium and silicon to the chemical enrichment of the ISM. Hence, the behaviour of these elements would be expected to follow each other in a galaxy that has experienced continuous or smooth star formation (see Pagel 1997; de Freitas Pacheco 1998). However, there is observational evidence for two separated bursts of star formation within the LMC. Both metallicity and age distributions are bimodal, viz. young, metal-rich clusters with ages less than 3-4 Gyr and old, metal-poor clusters $([\mathrm{Fe} / \mathrm{H}]<-1.5 \mathrm{dex})$ with ages between $10-$ 12 Gyr (see Hill et al. 2001). During the hiatus, the ISM has continued to have been enriched in iron via type I supernovae events - which could explain the observed low $\Delta[\mathrm{O} / \mathrm{Fe}]$ abundance ratio (see below). However, during the evolution of these low to intermediate mass stars - the ISM will also have been enriched in material ejected via stellar winds. During the giant phase, these stars experience dredgeup episodes that modify the photospheric CNO compositions. However, if deeper mixing occurs, the products of the $\mathrm{Ne}-\mathrm{Na}$ and $\mathrm{Mg}-\mathrm{Al}$ cycles (which occur at much hotter temperatures) may also be brought up to the stellar surface. This results in sodium and aluminium enhancements and the dilution of oxygen and magnesium in the photospheres of these stars (see, for example, Pagel 1997; Kraft et al. 1997). Hence, given the absence of massive star evolution for $\sim 6 \mathrm{Gyr}$ within the LMC, the evolution of low to intermediate mass stars and the $\mathrm{Mg}-\mathrm{Al}$ cycle may have become significant in modifying the relative amounts of $\mathrm{O}, \mathrm{Mg}$, Si replenished to the ISM.

\subsection{3. $\Delta[\alpha / \mathrm{Fe}]$ abundance ratio}

Unfortunately, it was only possible to derive an iron abundance for one LMC dwarf, viz, PS 34-16; hence, adopting this value as being representative of the global LMC ISM is dangerous. However, it is interesting to note that iron appears to be less deficient than the $\alpha$-elements $(\mathrm{O}, \mathrm{Mg}, \mathrm{Si})$. Indeed, our estimate of the $\Delta[\alpha / \mathrm{Fe}]$ abundance ratio of -0.14 dex is in excellent agreement with that obtained for evolved B-type stars, viz. $-0.16 \mathrm{dex}$ (see Korn et al. 2000). Additionally, Russell \& Dopita (1992) found a comparable iron deficiency, $\Delta[\mathrm{Fe} / \mathrm{H}]=-0.19 \mathrm{dex}$, for a sample of 9 F-type supergiants. However, more recent studies of F-type supergiants/Cepheids report abundance ratios of +0.09 dex (see Table 7). Several models of chemical evolution within the Magellanic Clouds have been proposed (see, for example, Russell \& Dopita 1992; Pilyugin 1996; Pagel \& Tautvaišienè 1998). Russell \& Dopita (1992) concluded that a steeper slope of the IMF was a critical factor in reducing the $[\alpha / \mathrm{Fe}]$ abundance ratio, by decreasing the relative number of massive stars, and therefore the relative contributions of type II to type I supernovae to the chemical enrichment of the LMC. By contrast, Pilyugin (1996) reproduced the low oxygen to iron abundance ratio by considering a model with enriched Galactic winds and episodic star formation. Several authors have proposed that dwarf galaxies experience bursts of star formation separated by long periods of quiescence (see Matteucci 1990; Gilmore \& Wyse 1991). Indeed, Pagel \& Tautvaišienė (1998) reproduced the deficit of oxygen relative to iron within the 
LMC using bursting star formation and without invoking selective winds. However, these models fail to reproduce the deficiencies of the other $\alpha$-elements (viz. $\mathrm{Mg}, \mathrm{Si}$ ) observed in the LMC.

It is clear that much more abundance information is required in order to constrain further evolutionary models of the chemical enrichment of the LMC. Detailed analyses of objects (stellar and nebulae) both young and old are needed to establish the homogeneity of abundance patterns throughout the Clouds and to constrain the age-metallicity relationship. Instruments such as FLAMES/GIRAFFE due to be commissioned on UT2 (ESO) present an efficient means of observing large numbers of objects within the Magellanic Clouds.

\section{Conclusions}

1. LMC membership of the 5 programme stars was confirmed using stellar radial velocities and the observation of interstellar $\mathrm{Ca} \mathrm{H}$ and $\mathrm{K}$ absorption features due to LMC gas in the stellar spectra.

2. Analysis of the B-type dwarfs infer a present-day LMC metallicity of $-0.31 \pm 0.04 \mathrm{dex}$; this is consistent with previous studies of F-type supergiants, Cepheids and H II regions.

3. No significant differences were found between the metallicity of cluster and field objects.

4. Two objects associated with the H II region LH 104 appear to be metal-rich compared with the global metallicity of the ISM. This may be evidence for localized chemical inhomogeneities within the LMC, as a result of a greater massive star formation.

5. Tentative evidence is provided for a lower oxygen to iron abundance ratio, viz. $\Delta[\mathrm{O} / \mathrm{Fe}] \sim-0.14$ dex. This is also observed in studies of more evolved B-type stars and is consistent with bursts of star formation separated with periods of quiescence.

6. Comparison of the $\Delta[\mathrm{Mg} / \alpha]$ abundance ratio in B-type stars, Cepheids and F-type supergiants provides evidence for a over-deficiency of this element. We postulate that deep mixing episodes, during the giant phase of low to intermediate mass stars, may contribute to this abundance pattern, in the absence of massive star formation for $\sim 6 \mathrm{Gyr}$ within the LMC.

Acknowledgements. We would like to thank the staffs of the AngloAustralian Observatory, Kitt Peak National Observatory and the South African Astronomical Observatory for their assistance in obtaining the observational data. Data reduction and analysis was performed on the PPARC funded N. Ireland STARLINK node. WRJR acknowledges financial assistance from the PPARC and CT is grateful for the financial support received from DEFHTE and the Dunville Scholarship's fund.

\section{References}

Andrievsky, S. M., Kovtyukh, V. V., Korotin, S. A., Spite, M., \& Spite, F. 2001, A\&A, 367, 605

Auer, L. H., \& Mihalas, D. 1972, ApJS, 24, 193

Barbuy, B., de Freitas Pacheco, J. A., \& Castro, S. 1994, A\&A, 283, 32
Becker, S. R., \& Butler, K. 1989, A\&A, 209, 244

Blaauw, A. 1961, Bull. Astr. Inst. Neth., 15, 265

de Freitas Pacheco, J. A. 1998, A\&A, 116, 1701

Dufour, R. J. 1984, IAU Symp., 108, 353

Dufton, P. L., Brown, P. J. F., Fitzsimmons, A., \& Lennon, D. J. 1990, A\&A, 232, 431

Dufton, P. L., McErlean, N. D., Lennon, D. J., \& Ryans, R. S. I. 2000, A\&A, 353, 311

Garnett, D. R. 1995, Rev. Mex. A\&A, 3, 141

Gies, D. R., \& Lambert, D. L. 1992, ApJ, 387, 673

Gilmore, G., \& Wyse, R. F. G. 1991, ApJ, 367, L55

Gummersbach, C. A., Kaufer, A., Schäfer, D. R., Szeifert, T., \& Wolf, B. 1998, A\&A, 338, 881

Hambly, N. C., Rolleston, W. R. J., Keenan, F. P., Dufton, P. L., \& Saffer, R. A. 1997, ApJS, 111, 419

Hardorp, J., \& Scholz, M. 1970, ApJ, 154, 1111

Henize, K. G. 1956, ApJS, 2, 315

Hilker, M., Richtler, T., \& Gieren, W. 1995, A\&A, 294, 648

Hill, V., Andrievsky, S., \& Spite, M. 1995, A\&A, 293, 347

Hill, V., François, P., Spite, M., Primas, F., \& Spite, F. 2001, A\&A, 364, L19

Howarth, I. D. 1983, MNRAS, 203, 301

Howarth, I. D., Murray, J., \& Mills, D. 1994, Starlink User Note, No. 50.15

Hubeny, I., Heap, S. R., \& Lanz, T. 1998, ASP Conf. Ser., 131, 108

Kaufer, A., Szeifert, T., Krenzin, R., Baschek, B., \& Wolf, B. 1994, A\&A, 289, 740

Kilian, J. 1994, A\&A, 282, 867

Korn, A. J., Becker, S. R., Gummersbach, C. A., \& Wolf, B. 2000, A\&A, 353, 655

Kovtyukh, V. V., \& Andrievsky, S. M. 1999, A\&A, 351, 597

Kraft, R. P., Sneden, C., Smith, G. H., et al. 1997, AJ, 113, 279

Kurucz, R. 1991, in Precision Photometry: Astrophysics of the Galaxy, ed. A. G. Philip, A. R. Upgren, P. L. Janes (L. Davis Press, Schenectady), 27

Larsen, S. S., Clausen, J. V., \& Storm, J. 2000, A\&A, 364, 455

Lennon, D. J., Brown, P. J. F., \& Dufton, P. L. 1988, A\&A, 195, 208

Lennon, D. J., Dufton, P. L., Mazzali, P.A., Pasian, F., \& Marconi, G. 1996, A\&A, 314, 243

Lucke, P. B. 1974, ApJS, 28, 73

Luck, R. E., Moffett, T. J., Barnes, T. G., \& Gieren, W. P. 1998, ApJ, 115,605

Luks, T. H., \& Rohlfs, K. 1992, A\&A, 263, 41

Massey, P. 1997, A User's Guide to CCD Reductions with IRAF, NOAO Laboratory

Massey, P., Valdes, F., \& Barnes, J. 1992, A User's Guide to Reducing Slit Spectra with IRAF, NOAO Laboratory

Matteucci, F. 1990, IAU Symp., 143, 625

Napiwotzki, R., Schönberner, D., \& Wenkse, V. 1993, A\&A, 268, 653

Olszewski, E. W., Suntzeff, N. B., \& Mateo, M. 1996, ARA\&A, 34, 511

Pagel, B. E. J. 1997, in Nucleosynthesis and Chemical Evolution of Galaxies (Cambridge University Press)

Pagel, B. E. J., \& Tautvaišienè, G. 1998, MNRAS, 299, 535

Parker, J. W., Garmany, C. D., Massey, P., \& Walborn, N. R. 1992, AJ, 103, 1205

Pilyugin, L. S. 1996, A\&A, 310, 751

Richtler, T., Spite, M., \& Spite, F. 1989, A\&A, 225, 351

Rolleston, W. R. J. 1991, Ir. Astron. J., 20, 60

Rolleston, W. R. J., Brown, P. J. F., Dufton, P. L., \& Howarth, I. D. 1996, A\&A, 315, 95 (RBDH96)

Rolleston, W. R. J., Dufton, P. L., Fitzsimmons, A., Howarth, I. D., \& Irwin, M. J. 1993, A\&A, 277, 10 
Rolleston, W. R. J., Dufton, P. L., McErlean, N. D., \& Venn, K. A. Stathakis, R. A., \& Johnston, H. M. 1997, The RGO Spectrograph 1999a, A\&A, 348, 728 Manual, AAO UM, 2.3

Rolleston, W. R. J., Hambly, N. C., Keenan, F. P., Dufton, P. L., \& Saffer, R. A. 1999b, A\&A, 347, 69

Rolleston, W. R. J., Smartt, S. J., Dufton, P. L., \& Ryans, R. S. I. 2000, A\&A, 363, 537

Russell, S. C., \& Bessell, M. S. 1989, ApJS, 70, 865

Russell, S. C., \& Dopita, M. A. 1990, ApJS, 74, 93

Russell, S. C., \& Dopita, M. A. 1992, ApJ, 384, 508

Schild, H., \& Testor, G. 1992, A\&AS, 92, 729

Shaver, P. A., McGee, R. X., Newton, L. M., Danks, A. C., \& Pottasch, S. R. 1983, MNRAS, 204, 53

Shobbrook, R. R., \& Visvanathan, N. 1987, MNRAS, 225, 947

Spite, F., Richtler, T., \& Spite, M. 1991, A\&A, 252, 557

Testor, G., \& Niemela, V. 1996, Proc. 11th IAP Meet., 487

Testor, G., \& Niemela, V. 1998, A\&AS, 130, 527

Tody, D. 1986, IRAF User Manual, NOAO Laboratory

Valdes, F. 1992, A Guide to the Slit Spectra Reduction Task DosLit, NOAO Laboratory

Vidal, C. R., Cooper, J., \& Smith, E. W. 1973, ApJS, 25, 37

Wayte, S. R. 1990, ApJ, 355, 473

Westerlund, B. 1997, in The Magellanic Clouds (Cambridge University Press)

Willmarth, D., \& Barnes, J. 1994, A User's Guide to Reducing Echelle Spectra with IRAF, NOAO Laboratory 